\title{
La pobreza como categoría moral. Por qué la riqueza no es suficiente para dejar de ser pobre
}

La pauvreté comme catégorie morale. Ou pourquoi la richesse n'est pas suffisante pour cesser d'être pauvre

Poverty as a moral category or why wealth is not sufficient to stop being poor

\section{Pascale Absi}

\section{(2) OpenEdition}

Journals

\section{Edición electrónica}

URL: http://journals.openedition.org/bifea/7675

DOI: $10.4000 /$ bifea.7675

ISSN: 2076-5827

\section{Editor}

Institut Français d'Études Andines

\section{Edición impresa}

Fecha de publicación: 1 diciembre 2015

Paginación: 415-413

ISSN: 0303-7495

\section{Referencia electrónica}

Pascale Absi, « La pobreza como categoría moral. Por qué la riqueza no es suficiente para dejar de ser pobre », Bulletin de l'Institut français d'études andines [En línea], 44 (3)| 2015, Publicado el 08 diciembre 2015, consultado el 10 diciembre 2020. URL : http://journals.openedition.org/bifea/7675 ; DOI : https://doi.org/10.4000/bifea.7675

\section{(a) $\mathbb{Q} \Theta \Theta$}

Les contenus du Bulletin de l'Institut français d'études andines sont mis à disposition selon les termes de la licence Creative Commons Attribution - Pas d'Utilisation Commerciale - Pas de Modification 4.0 International. 


\title{
La pobreza como categoría moral. Por qué la riqueza no es suficiente para dejar de ser pobre
}

\author{
Pascale Absi*
}

\section{Resumen}

Una de las razones dadas para explicar la pobreza es la supuesta incapacidad de los pobres para administrar adecuadamente su dinero. En numerosos países, proyectos de educación financiera proponen remediar este «problema». Más allá de saber cómo los pobres gestionan su dinero y si lo hacen de manera eficiente, este artículo propone una reflexión sobre los prejuicios que vehiculan los postulados de la educación financiera así como de algunos académicos y opiniones populares, cuando se refieren a las prácticas financieras de determinados grupos de la población. A través del ejemplo de los mineros y de las prostitutas de Bolivia, se muestra que su relación con el dinero es concebida como el síntoma de un desajuste con los valores hegemónicos de la civilización. Atrapada en paradigmas culturalistas y morales, la sospecha de irracionalidad económica termina por encerrar a los pobres en su condición subalterna, independientemente de su situación económica. Se vuelve una estrategia de dominación. Es por eso que, aun cuando se capitalizan y consumen como los ricos (o sea aun cuando tienen una economía exitosa), algunas personas no pueden liberarse del estigma. Es el caso de los trabajadores de las cooperativas mineras cuyos procesos de ascensión social pueden ser alienados por su asignación a la llamada «cultura de la pobreza». Es también el caso de las prostitutas cuando la imposibilidad moral de pensar la prostitución como una actividad rentable y, por lo tanto, como una estrategia eficaz, contribuye a descalificarlas.

Palabras clave: dinero, educación financiera, moral, pobreza

* Antropóloga, Instituto de Investigación para el Desarrollo (IRD), UM R CESSM A, Programa ANR Suds II 2011-2015 «Crise, travail, emploi et revenus dans des sociétés des Suds ». 


\title{
La pauvreté comme catégorie morale. 0 u pourquoi la richesse n'est pas suffisante pour cesser d'être pauvre
}

\author{
Résumé
}

L'une des raisons invoquées pour expliquer la pauvreté est l'incapacité supposée des pauvres de gérer leur argent. Dans de nombreux pays, des programmes d'éducation financière sont mis en place pour remédier à ce «problème ». Au-delà de savoir comment les pauvres gèrent leur argent et si ils le font de manière efficace, cet article propose de réfléchir aux préjugés véhiculés par les postulats de l'éducation financière mais aussi par des chercheurs et des opinions populaires, lorsqu'ils se réfèrent aux pratiques monétaires de certains groupes de populations. À travers l'exemple des mineurs et des prostituées de Bolivie, nous montrerons que leur relation avec l'argent est conçue comme le symptôme d'une inadéquation avec les valeurs hégémoniques de la civilisation. Attrapé dans des paradigmes culturalistes et moraux, le soupçon d'irrationalité économique finit par enfermer les pauvres dans leur condition subalterne, indépendamment de leur situation économique. Elle devient une stratégie de domination. $C^{\prime}$ est la raison pour laquelle, même lorsqu'ils se capitalisent et consomment comme des riches (c'està-dire lorsqu'ils connaissent des succès économiques), certaines personnes ne peuvent se libérer du stigmate. C'est le cas des travailleurs des coopératives minières dont les processus d'ascension sociale peuvent même être aliénés par leur assignation à ce que l'on a appelé « la culture de la pauvreté ». C'est également le cas des prostituées car l'impossibilité morale à penser la prostitution comme une activité rentable et, donc, une stratégie efficace, contribue à les déqualifier.

Mots-clés : argent, éducation financière, morale, pauvreté

\section{Poverty as a moral category or why wealth is not sufficient to stop being poor}

\begin{abstract}
One of the reasons given to explain poverty is the supposed inability of poor people to manage their money properly. Many countries have introduced financial education projects in an attempt to remedy this "problem". Rather than seeking to find out how poor people manage their money and whether they do so efficiently, this article reflects on the prejudices underlying the premises of financial education, as well as some academic arguments and popular opinions with regard to the financial practices of certain groups of people. Using miners and prostitutes in Bolivia as examples, the article shows that their relationship with money can be seen as symptomatic of a mismatch with the hegemonic values of civilization. Caught up in culturalist and moral paradigms, the suspicion of economic irrationality ultimately traps the poor in their subaltern status, regardless of their economic situation, and becomes a strategy of domination. This is the reason why, even when they accumulate capital and consume in the same way as rich people (in other words, even when they are economically successful), some people are unable to escape from being stigmatized. Workers in mining cooperatives are a case in point, as their social ascent may be thwarted by being assigned to what used to be known as the "culture of poverty". Prostitutes are a similar example, as the moral impossibility of thinking of prostitution as a profitable activity and, therefore, an effective strategy, contributes to their being discredited.
\end{abstract}

Keywords: money, financial education, morals, poverty 
Una de las razones dadas para explicar la pobreza es la supuesta incapacidad de los pobresı para administrar adecuadamente su dinero. La falta de previsión, de planificación y de ahorro, los gastos irracionales, el endeudamiento incontrolado forman parte del arsenal explicativo de algunos expertos en desarrollo (Guérin, 2012; 2014). Motivan, tanto en Bolivia como en otras partes, la implantación de programas de capacitación financiera, a menudo vinculados con la concesión de microcréditos. Así, en 2013, en virtud de la lucha contra la pobreza, la «educación económica y financiera» se ha incorporado en los programas escolares bolivianos. La creencia en el analfabetismo financiero de los pobres no es algo nuevo. Ya orientaba los actos de caridad y de beneficencia en el siglo XVIII (Belliar, 2011). Tampoco es exclusiva del ámbito del desarrollo, de los banqueros 2 o de los filántropos: la crítica infunde también a menudo la visión que los grupos de población que escapan a esta categorización tienen de sus conciudadanos pobres. La división entre buenos y malos gestores - tal como la entiende el capitalismo dominante- no se refiere solo a lo contable. La idea de que los pobres no saben administrar sus recursos suele estar acompañada de la que no poseen los valores que les permitirían tomar las buenas decisiones respecto a gastos, deuda o ahorro. Su relación con el dinero sería solo uno de los síntomas de desajuste cultural y moral con los valores hegemónicos de la civilización. De este modo, la crítica del analfabetismo financiero se dirige de manera más general a las opciones de vida de algunos grupos de la población, ocultando los factores estructurales de la pobreza. Es más fácil criticar a los pobres que remediar a la insuficiencia de sus ingresos.

Sin embargo, numerosos estudios han demostrado que los pobres y los analfabetos desarrollan estrategias, modos de cálculo y de ahorros sofisticados y eficientes (Lelart, 1990; Rutherford, 2001; M oulévrier y Lazuech, 2008; Daryl et al., 2009; Absi, 2009; etc.). Muchos enfrentan los riesgos de la vida, y algunos logran enriquecerse gracias a estas tácticas. Es el caso de los mineros cooperativistas y de las prostitutas de Potosí, en Bolivia, con los cuales he llevado a cabo unas investigaciones antropológicas a lo largo de las dos últimas décadas. Se requiere menos tiempo para escuchar, reiteradamente, comentarios de otros sectores de la población sobre la incapacidad de los «pobres» para sacar provecho de sus ganancias. Lo que me interesa investigar en este artículo no es tanto cómo los mineros y las prostitutas gestionan su dinero, sino por qué, a pesar de sus logros - 0 sea aun cuando ganan plata y se capitalizan-, no logran escapar a su categorización como agentes irracionales. Me propongo emprender aquí una reflexión sobre los prejuicios que vehiculan algunos discursos populares e incluso de investigadores, y los postulados de la educación financieras cuando se refieren a las prácticas económicas de determinados grupos de la población. Encerrada en paradigmas culturalistas y morales, la sospecha de irracionalidad económica

1 Empleo el término «pobre» por referencia a las categorización de los programas de lucha contra la pobreza, no como una categoría social coherente.

2 En Francia, Pascale Moulevrier y Gilles Lazuech (2008) han analizado cómo la percepción de los clientes «pobres» como agentes económicos irracionales incide en el comportamiento de los banqueros frente a ellos. 
termina por atrapar a los pobres en su condición subalterna, independientemente de su situación económica. Es por esta razón que, aun cuando se vuelven ricos (y consumen como los ricos), algunas personas no pueden liberarse del estigma. El caso de los trabajadores de las cooperativas mineras de Potosí es un buen ejemplo de cómo la construcción moral de la pobreza puede llegar a alienar procesos de ascenso social. En cuanto a las prostitutas, veremos que la imposibilidad moral de pensar la prostitución como una actividad rentable y, por lo tanto una estrategia eficaz, contribuye a descalificarlas.

\section{LA PO BREZA COMO CATEGO RÍA MO RAL}

Más allá de su eficiencia, las estrategias de los pobres para administrar su dinero «escapan de las categorías etnocéntricas y economicistas» (Guérin, 2012: 51). Por ello, muchos de los beneficios de la circulación monetaria permanecen invisibles a los ojos de los postulados del pensamiento económico puro (el prestigio, el placer, los lazos sociales de la deuda, etc.). Sin embargo, estos no necesariamente contradicen la lógica capitalista. Algunas prácticas asociadas al derroche, como los gastos ostentosos y la magnanimidad, que obligan a los beneficiarios a devolver con prestaciones en servicios o en especie, pueden responder a un buen cálculo financiero. Así, es al financiar los rituales que los socios de las cooperativas mineras aseguran su poder sobre los yacimientos y la mano de obra (Absi, 2005). En otros contextos, investigaciones recientes han analizado el rol que juegan en las ciudades bolivianas los gastos suntuosos - como los que se realizan en la fiesta del Gran Poder en La Paz (Tassi, 2010; Tassi et al., 2013) - en la creación de redes de alianza y de obligaciones recíprocas movilizadas por las actividades comerciales de los participantes. Como lo expresa Isabelle Guérin (2014: 145):

En contextos en los cuales invertir en la 'riqueza social' es tan esencial como acumular bienes o patrimonio financiero, la separación entre consumo, inversión y ahorro se vuelve difusa y finalmente parece muy arbitraria pues cada préstamo/don debe ser reembolsado o devuelto.

Sin embargo, el análisis de las lógicas financieras de los pobres y de sus resultados a largo plazo resulta bastante impotente frente a los prejuicios que transmite el discurso de algunos expertos. Así, Isabelle Guérin (2014) reporta ciertos análisis psicologistas que explican la ausencia de ahorro por el hecho de que los pobres serían consumidores impulsivos e impacientes que viven en la inmediatez. Ella relata incluso los esfuerzos de algunos economistas para calcular los ahorros que un vendedor de verduras de la India realizaría al beber dos tazas de té menosal día. En Bolivia, los gastos en fiestas y el consumo de los mineros y de los comerciantes siguen siendo señalados con el dedo - también por la prensa- como un síntoma de frivolidad casi congénita ligada a su origen campesino e indígena, y no como la evidente prueba de un manejo eficaz. De hecho, el origen de su fortuna se remite sistemáticamente a factores externos, como los precios favorables de los minerales o la permisividad del Estado frente al contrabando. En pocas palabras, los pobres son pobres debido a su comportamiento, ipero, si se enriquecen nunca se reconoce que es también gracias a este! 
De este modo, los fundamentos del discurso académico sobre la irracionalidad económica se cristalizan en un discurso popular que suprime los factores estructurales de la pobrezay acaba naturalizándola. Interpretada como el resultado de una predisposición psicocultural, se convierte en una categoría esencialista desligada de los procesos sociales, también del posible enriquecimiento de los pobres. Hace ya más de veinte años que Michel Agier (1995; 1989) - después de otros sociólogos como Anthony Leeds (1971) - denunció los avatares de este oxímoron en la literatura antropológica, que ha dado origen al concepto de «cultura de la pobreza» de la pluma de O scar Lewis (1969), a su etnificación (Herskovits, 1943) y, de manera más amplia, a la confusión entre precariedad social y cierto tipo de pensamiento marginal. Los ejemplos anteriores muestran que a pesar de varias décadas de críticas, estos axiomas no han desaparecido del campo académico, menos aún de las exégesis populares.

En Bolivia, los comentarios despectivos sobre la frivolidad y el consumismo de sus ocupantes, que a veces suscita la visión de una antena parabólica sobre una vivienda que se interpreta como «pobre», confirman la evidente dimensión moral del juicio. El supuesto pobre debería cultivar la miseria, que es precisamente la fuente de su marginalidad y estigmatización. D e hecho, la prudencia, la anticipación y la parsimonia preconizadas por los programas de educación financiera son virtudes más que lógicas de cálculo económico. Desde su inicio, los programas de educación financiera se presentaron como una «reeducación moral». Ya en el siglo XVIII europeo, los filántropos inculcaban a los desfavorecidos no solo formas de gestión financiera, sino también normas de comportamiento (Belliar, 2011). M ientras que los «buenos» pobres eran susceptibles de ser recuperados para convertirse en miembros útiles de la sociedad, la represión se abatía sobre los «malos» (trabajos forzados, confinamiento) (Belliar, 2011). Existían manuales que indicaban cómo distinguir los unos de los otros. Publicado en 1820 por el pedagogo y filántropo De Gérando, Le Visiteur du pauvre se presenta como una guía de investigación y acción destinada a la comprensión y la curación de las enfermedades morales que sufrirían los pobres (De Gérando 1989 [1820]. Es significativo que su autor fuera también el creador de uno de los primeros métodos de investigación etnográfica, publicado en el año 1800 por la «Société des O bservateurs de l'H omme» de París (Chappey et al., 2014). Entre la cultura del «salvaje» y la cultura del pobre hay solo un paso.

La interpretación de la pobreza como un síntoma de fracaso cultural y moral es un buen argumento para los Estados. Legitima la tutela, incluso la inferioridad cívica, de ciertos grupos de la población. De este modo, el criterio de la adecuación - 0 la no adecuación- a los valores y las normas de comportamiento requeridas por el capitalismo de mercado interviene en la reproducción de las jerarquías sociales. Por lo tanto, sirve a los intereses de las clases dominantes. En Bolivia, la acusación moral a los pobres es un mecanismo central de descalificación del ascenso social de personas de origen campesina e indígena que amenazan la posición de las élites tradicionales (pensadas como blancas o mestizas). 


\section{EL ILEGÍTIMO ASCENSO SO CIAL DE LOS MINEROS DE POTOSÍ}

Como se desprende de los escritos del cruceño Gabriel René M oreno, durante el siglo que siguió a la independencia de Bolivia en 1825, la supuesta irracionalidad económica de las poblaciones indígenas fue construida como un obstáculo para su participación en el desarrollo de la Nación y por tanto su acceso a la ciudadanía. De esta manera, el sufragio se mantuvo masculino, censitario y reservado a los letrados (y como consecuencia vedado a las masas indígenas) hasta 1952. Hoy en día, el argumento de la irracionalidad económica segrega a la sociedad sobre la base de una categorización moral que distingue las élites tradicionales de los «advenedizos» («desclasados» según la glosa popular), cuyos patrones de consumo estigmatizan: los gastos festivos, de equipamiento (vehículos, electrónica, electrodomésticos)... «Tienen una tele último modelo pero no saben alimentarse», «una movilidad "full equipada", pero no saben manejar», «en vez de hacer hijos de manera desconsiderada - incluso adúltera - harían mejor en pensar en su educación» son comentarios que se suelen escuchar. Con calificativos como «impropio», «ostentoso» 0 «contrapuesto» a lo que deberían ser las prioridades familiares y una planificación razonable, los juicios emitidos por las clases altas sobre su modo de vida y de consumo encierran a determinados grupos de la población en una alteridad que dificulta la consolidación de la ciudadanía sobre la base de individuos iguales por naturaleza, independientemente de su condición social, étnica o de género3. Hacerse rico es tanto una cuestión de legitimidad y de autorización social como un proceso económico.

Durante décadas, la imagen de los trabajadores de las cooperativas mineras de Bolivia se fue consolidando en torno a la tríada «pobres, indios e ignorantes», cuyos términos son intercambiables. Ha alimentado la visión - entre la compasión y el desprecio- del resto de la sociedad, de la literatura 4 y de los medios de comunicación. Por mucho tiempo los mineros han ejemplificado una versión nacional de la «cultura de la pobreza». Pero, en el transcurso de la década de 2000, el ascenso de los precios de los minerales trastornó el panorama. Los ingresos de los mineros cooperativistas de Potosí, remunerados por la venta de su producción, se multiplicaron, a veces de forma exponencial. La ciudad cambió de cara: centenares de casas de varios pisos reemplazaron a las viejas casonas de adobe, galerías comerciales y hoteles de cinco estrellas surgieron gracias al dinero de la mina. Los mineros también compraron autos, lujosos $4 \times 4$ y minibuses. Las bodas, los bautizos, las celebraciones a los santos y los rituales mineros tragaron

3 Juicios algo parecidos fueron recogidos por Jean-François Laé et Numa Musard (1985), en su obra sobre los habitantes de los suburbios pobres de una ciudad francesa, considerados agresivos, despilfarradores, desagradecidos con sus benefactores. Sin embrago, los autores demuestran cómo las actitudes que da pie a estos comentarios responden a una lógica personal y colectiva que organiza de manera estratégica y solidaria la vida económica y social de estos barrios.

4 Pienso, por ejemplo, en la novela de Jaime Mendoza, En las tierras de Potosí, publicada en 1911 y reimpresa varias veces hasta el día de hoy, y presente en todas las ferias de libros. 
montos considerables. En 2007, durante el Carnaval, los cooperativistas «secaron» la fábrica de cerveza de Potosí. La mina atrajo a muchos migrantes provenientes de otras ciudades y de las comunidades campesinas indígenas de los alrededores. Después de bordear por mucho tiempo los 120000 habitantes, en medio del boom minero la ciudad habría alcanzado una población de unos 1600005. Obviamente no todos los mineros se han hecho millonarios; pero en mayor 0 menor medida todos han participado en la carrera por el consumo.

La extraordinaria afluencia de dinero desembocó en una crisis social y de representaciones sin precedente (Absi, 2010). La llegada de esos nuevos ricos alteró la jerarquía, en las que los mineros, considerados «indígenas, pobres, y rústicos», debían ocupar los peldaños más bajos. Los mineros, antes relegados a los barrios mineros, invadieron los espacios de la pequeña burguesía urbana. Frecuentaban los mismos restaurantes, las mismas escuelas y sus autos se disputaban las mismas calles embotelladas. Los trabajadores del subsuelo fueron recalificados de indígenas pobres a nuevos ricos. Sus gastos «irracionales» se volvieron el argumento para deslegitimizar su ascenso social y sus reivindicaciones políticas.

Las acusaciones de los habitantes de Potosí pertenecientes a las clases medias y altas y los ataques de la prensa local contra los cooperativistas pusieron de manifiesto la amenaza que representó su enriquecimiento en una sociedad que se piensa al mismo tiempo en términos socioeconómicos, culturales y raciales. «ndios refinados», «nuevos ricos mal pulidos» son algunas de las ideas que se hacen los citadinos de pura cepa de los cooperativistas, a los que les reprochan haber adoptado los aspectos más ostentosos de la civilización urbana sin conocer su etiqueta ni sus códigos. Se les acusó de fomentar la inflación, de tener grandes autos sin saber conducir, de perturbar la tranquilidad de los vecinos apropiándose de los espacios de la burguesía local, de desfigurar la ciudad con sus casas, de hundir la sociedad en el alcoholismo, la prostitución y el desorden de los dinamitazos, incluso de no esperar su vuelto en el mercado...; i en resumen, de no saber «lo que es el dinero». La prensa tampoco dudó en multiplicar los titulares edificantes: «Mineros se farrean la bonanza minera» 0 «El poder cooperativista atemoriza a la ciudadanía»6. La ausencia de ahorro fue, junto con la violencia familiar y el adulterio, el principal argumento de su ilegitimidad7.

Lo más sorprendente es que los mineros mismos empezaron a asumir esos estigmas. Muchos pensaron que la embriaguez del dinero perturbaría el funcionamiento de una sociedad que, al tiempo de alentar el éxito nacido del

5 Actualidad de la minería, Fencomin, La Paz, 02/07/07.

6 El Potosí, 04/09/2007; La Razón, La Paz, 02/07/2002.

7 Esta ausencia de ahorro se puede explicar por razones objetivas. El ahorro es, en primer lugar, una forma de gestión de la carencia que corresponde a las mujeres. A la inversa, la gestión del boom fue sobretodo asunto de los hombres. Hasta la compra de un terreno o de un auto dependieron de un ingreso excepcional o de una acumulación a muy corto plazo (algunas semanas). Las alzas inflacionarias de las décadas pasadas, que arruinaron a muchos mineros, también indujeron la 
esfuerzo individual, condena el enriquecimiento brusco, fuente de desigualdades demasiado explícitas. En la lógica minera, esta interpretación se traduce en la idea de que los mineros tentados por el diablo y el poder del dinero se han sumergido en una vida de derroche y de transgresiones. Asimismo, pendientes de la mirada reprobadora de las clases superiores que no los aceptan en sus filas, los mineros han juzgado su éxito y alienado sus propios proyectos de ascenso social. Casi con alivio, con el sentimiento de retornar al orden, han visto venir la crisis del 2008 y luego la estabilización de los precios de los minerales a niveles no tan altos.

\section{PARA SER ACEPTABLE, UNA PROSTITUTA DEBE SER PO BRE}

Al igual que los mineros, la salida de la pobreza extrae a las prostitutas de la posición que les otorga el imaginario social. Solo la miseria podría justificar el comercio sexual: para ser moral, la prostituta tiene que ser pobre. Este axioma influye, a menudo inconscientemente, en los trabajos de los investigadores. El hecho de que las prostitutas dilapidarían su dinero es un postulado corriente en los estudios sobre la prostitución en las diversas regiones del mundo. Un breve repaso sugiere, sin embargo, que la mayoría está menos interesada en cómo las mujeres administran su dinero y qué hacen con él que en recalcar su más singular destino: cuando las prostitutas derrochan el dinero.

Si se observan entre las prostitutas bolivianas prácticas que pueden ser interpretadas como «dilapidación», por ejemplo cuando los domingos pagan muchas rondas en los bares o cuando acumulan ropa que en parte nunca se van a poner. Pero estos comportamientos no caracterizan el conjunto de sus gastos. La focalización en este aspecto y el poco interés por otras inversiones parecen corresponder a un sesgo ideológico. Como en el caso de los mineros, el modo en que se perciben los patrones de consumo de las prostitutas está marcado por consideraciones morales. Refleja la ilegitimidad que se atribuye a la venta de servicios sexuales, así como cierto modo de pensar sobre lo que debería ser el patrón de consumo correcto de una mujer, más aún de una mujer pobre. En este caso, a las conminaciones morales de género se añaden aquellas relacionadas con la posición socioeconómica.

Los motivos mencionados con más frecuencia para explicar la dilapidación son psicológicos. Dilapidar rápidamente el dinero de la prostitución, al borrar la mancha de la sexualidad venal permitiría blanquearlo y compensar con el disfrute compulsivo el peso de sus orígenes turbios. Estudios realizados en Francia (por ejemplo Deschamps \& Canarelli, 2008) muestran que esta interpretación se comparte a veces con las mismas prostitutas. En Bolivia, en cambio, la idea de que el dinero de la prostitución estaría ensuciado por la venta de servicios sexuales y que habría que deshacerse de él lo más rápido posible, es desconocida (Absi, 2014).

elección de las inversiones en bienes. El otro defecto del ahorro es que invisibiliza la riqueza, en una configuración cultural en la que la magnanimidad, la ostentación y el prestigio forman un sistema (Absi, 2010). 
Sin ser falsa, la interpretación simbólica de la volatilidad de las ganancias de la prostitución escamotea un parámetro más concreto: se trata exclusivamente de dinero en efectivo obtenido día a día, por no decir minuto a minuto. Al igual que en el caso de los mineros cooperativistas, la ausencia de ingresos fijos hace que el gasto sea menos doloroso en tanto una nueva ganancia puede compensarlo un momento después. La esperanza de que esta noche la suerte esté con ellas incita a las prostitutas a pensar que recuperarán rápidamente el dinero gastado. Gastar es también una manera de actualizar su motivación, de seguir movilizadas. Además, llevar una vida inestable cuesta caro. Las mujeres con frecuencia cambian de ciudad, deben comer y divertirse fuera y por tanto en sociedad, lo que conlleva invitaciones recíprocas. Crear una deuda social en un contexto en el que la única seguridad jurídica es la de la red social no es tampoco un cálculo irracional.

Sin embargo, más allá de los gastos visibles, gran parte del dinero se envía a las familias para el sustento de los hijos, la construcción de una casa, la mejora de la vivienda de los padres o la participación en el capital de una empresa familiar. Estos gastos favorecen una nueva posición y un nuevo estatus dentro de las familias, quienes fingen no conocer el origen de estos ingresos. $Y$ al final, los gastos de las prostitutas bolivianas no resultan ser muy «ostentosos». Sus primeras adquisiciones ponen de manifiesto sus frustraciones como jóvenes y el proyecto de independencia en el que a menudo se inscribe la entrada a la prostitución: ropa nueva en vez de la heredada de los mayores, una cama, un armario, un televisor, una cocina... Las mujeres no frecuentan los restaurantes ni las tiendas elegantes de los centros de la ciudad, sus hijos asisten a colegios públicos y ellas compran sus cosméticos a crédito a vendedores ambulantes. Finalemente, las prostitutas se comportan como cualquier persona de su clase que mejora sus ingresos. Sus gastos solo aparecen como excesivos en relación con la parsimonia de los «pobres»: la parsimonia, real, dictada por la escasez, pero también aquella que la moral asigna a los más pobres.

En el caso de las prostitutas, la crítica a su consumo ostentoso está ligada a la interpretación de que, para ser moral, es decir, no viciosa, la prostitución debe estar motivada por la extrema pobreza. U na vez obtenidos los primeros beneficios, las mujeres deberían pensar únicamente en salir de la prostitución y dedicar todas sus ganancias a su reconversión. De lo contrario, dejan de ser víctimas respetables para convertirse en culpables. Este planteamiento oculta que la pobreza extrema no es el único motivo de la entrada en prostitución. Esta puede permitir enfrentar un golpe duro, pero no todas las prostitutas están desesperadas. De hecho, la mayoría de ellas no provienen de los medios más pobres ni de los menos educados. Más que la pobreza, es la frustración frente a un mercado laboral que no cumple con sus promesas lo que motiva a las mujeres, a menudo con bachillerato, a dar el paso. Reconocer ello equivale a atribuir a la entrada en la prostitución cierta racionalidad dentro de un proyecto de ascenso social.

A la parsimonia moral del pobre que estigmatiza a los mineros que se han hecho ricos se añade la que se espera de las mujeres. Pese a todo, gastar su dinero en 
un bar o dilapidar los ingresos del hogar son comportamientos que siguen siendo concebibles si se trata de un hombre. Este no constituye la única infracción de género de las prostitutas en relación a sus gastos. Además, ellas se desligan de varias tareas que corresponden tradicionalmente a las mujeres: contratan a niñeras para sus hijos, a lavanderas para su ropa y sus cuartos, comen afuera en vez de cocinar, etc. Estas prácticas aparecen como un lujo en un entorno en el que las mujeres suelen ser más empleadas que patrones. Alimentan la idea de que las prostitutas son mujeres perezosas y ambiciosas que se niegan a conformarse con el sueldo de una trabajadora doméstica o con los ingresos de un esposo, y a asumir sus deberes conyugales y domésticos. Ya por ello merecerían el calificativo de «puta», el cual, como lo ha demostrado Gail Pheterson (2010) no se refiere únicamente a las mujeres que venden servicios sexuales, sino a cualquiera que infringe las reglas hegemónicas de la división sexual del trabajo y, por tanto, la dimensión de género del dinero. Así, las prostitutas aparecen doblemente culpables: como pobres y como mujeres; y se obvia el hecho que derrochar su dinero como un rico y como un hombre es una manera de salir del confinamiento subjetivo de la pobreza y del género.

\section{CONCLUSIÓ N: LA RED ENCIÓN A TRAVÉS DEL ASCETISMO}

La sospecha de irracionalidad económica - entendida como un conjunto de actitudes y valores arraigados en la cultura y la psicología de los pobres, transmitido de generación en generación, la cual «explica» su situación- condena a estos a la marginalidad. Frente a esta interpretación, incluso una mejoría en su condición no es suficiente para resolver su supuesta falta de adecuación a las normas sociales dominantes, en particular respecto a sus gastos y a pesar de que estos ponen de manifiesto el hecho de que los pobres no son tan pobres. Aquí vemos claramente la naturaleza contradictoria de las conminaciones a los que están sujetos los pobres: se les insta a salir de la pobreza, pero se les prohíbe usar los resortes del consumo, que distingue hoy en día a los marginados de los ciudadanos plenos (Hours, 2012). El pobre solo debe consumir para sobrevivir. De lo contrario, es percibido, en el mejor de los casos, como un ser irracional y, en el peor, como un falso pobre, un aprovechador (si su condición motiva la ayuda institucional). Cuando la pobreza funciona como una categoría moral, la salida no pasa pues por el enriquecimiento, sino por la redención mediante el ascetismo. 
La pobreza como categoría moral. Por qué la riqueza no es suficiente para dejar de ser pobre

\section{Referencias citadas}

ABSI, P., 2005 - Los ministros del diablo. El trabajo y sus representaciones en las minas de Potosí, xx + 339 pp.; La Paz: IFEA, IRD, PIEB, Embajada de Francia en Bolivia

ABSI, P., 2009 - Trabajo, género e ingresos entre las comerciantes minoristas de Potosí. T'inkazos, n. ${ }^{\circ}$ 26: 69-90; La Paz.

ABSI, P., 2010 - La parte ideal de la crisis. Los mineros cooperativistas de Bolivia frente a la recesión. Cuadernos de antropología social, $\mathbf{n}^{\circ}$ 31: 33-54; Buenos Aires.

ABSI, P., 2014 - De la subversion à la transgression. La valeur de l'argent dans les maisons closes de Bolivie. In: L'échange économico-sexuel (C. Broqua \& C. Deschamps, eds.): 61-88; París : Editions de l'EHESS.

AGIER, M., 1989 - Pauvreté, culture et exclusion. La question du sens en anthropologie urbaine. In: Villes du sud : sur la route d'Istanbul (E. Le Bris, ed.) : 227-243; París: ORSTOM. http://horizon.documentation.ird.fr/exl-doc/pleins_textes/ divers11-10/010005826.pdf (consulté le 16/02/2015).

AGIER, M ., 1995 - Pour une anthropologie critique de la pauvreté. Contribution au séminaire Le développement peut-il-être social ?, 21 pp. ; Royaumont: O RSTOM, MAE.

BELLIAR, C., 2011 - La charité est la vocation d'une dame ; le soin des pauvres est sa profession. Hannah More, 1745 - 1833. In: Prendre soin de soi, prendre soin d'autrui. Journée d'étude, Université de Tours, 2011. https://halshs.archivesouvertes.fr/hal-00842109 (consulté le 08/09/2014).

CHAPPEY, J.-L., CHRISTEN, C. \& M OU LLIER, I., 2014 - Joseph-Marie de Gérando (17721842). Connaître et réformer la société, 341 pp.; Rennes: Presses Universitaires de Rennes.

DARYL, C., MORDUCH, J., RUTHERFORD, S. \& RUTHVEN, 0., 2009 - Portfolios of the Poors. How the World's Poor Live on $\$ 2$ a Day, ix +283 pp.; Princeton: Princeton U niversity Press.

DE GÉRAND O, M.-J., 1989 [1820] - Le Visiteur du pauvre, xi + 546 pp.; París: Jean-M ichel Place.

DESCHAMPS, C. \& CANARELLI, P., 2008 - La fabrique de la passe. Sociétés. Revue des sciences humaines et sociales, $\mathbf{n} .^{\circ} \mathbf{9 9}:$ 47-60.

EL POTOSÍ, 2007 - 04/09/2007.

FENCO MIN, 2007 - Actualidad de la minería; La Paz, 02/07/07.

GUERIN, I., 2012 - L'éducation financière où comment apprendre aux pauvres à bien consommer. In: À quoi et comment dépenser son argent ? Hommes et femmes face aux mutations globales de la consommation (I. Guérin \& M. Selim, eds.): 51-71; París: L'H armattan.

GUERIN, I., 2014 - La microfinance et ses dérives. Emanciper, discipliner ou exploiter ?, 287 pp. ; París: Éditions Demopolis.

HERSKOVITS, M., 1943 - The Negro in Bahia, Brazil: a problem of method. American Sociological Review, vol. 8: 394-402.

HOURS, B., 2012 - La gouvernance par la consommation. In: À quoi et comment dépenser son argent ? Hommes et femmes face aux mutations globales de la consommation (I. Guérin \& M. Selim, eds.): 41-50; París: L'H armattan.

LEEDS, A., 1971 - The concept of the "culture of poverty": conceptual, logical, and empirical problems, with perspectives from Brazil and Peru. In: The Culture of Poverty: A Critique (E. B. Leacock, ed.): 226-286; New York, N.Y.: Simon and Schuster. 
LAE, J.-F. \& MUSARD, N., 1985 - L'argent des pauvres. La vie quotidienne en cité de transit, 209 pp.; París: Le Seuil.

LA RAZÓ N, 2002 - La Paz, 02/07/2002.

LELARD, M. (dir.), 1990 - La tontine : Pratique informelle d'épargne et de crédit dans les pays en voie de développement, vi + 356 pp.; London-Montrouge: John Libbey Eurotext.

LEWIS, O., 1969 - La Vida. Une famille porto-ricaine dans une culture de pauvreté : San Juan et New York, 819 pp.; París: Gallimard.

MEN DO ZA, J., 1911 - En las tierras de Potosí, 355 pp.; Barcelona: Tasso.

MOULÉVRIER, P. \& LAZUECH, G., 2008 - Les usages sociaux de l'argent : les « démunis » ont-ils quelque chose à nous apprendre ? In: L'argent des anthropologues, la monnaie des économistes (E. Baumann, L. Bazin, P. O uld-Ahmed, P. Phélinas, M. Selim \& R. Sobel, eds.): 55-71; París: L'Harmattan.

PHETERSO N, G., 2010 - El prisma de la prostitución, 190 pp.; Madrid: Talasa.

RUTHERFORD, S., 2001 - The Poor and their Money, viii + 121 pp.; Oxford: Oxford University Press.

TASSI, N., 2010 - Cuando el baile mueve montañas. Religión y economía cholo-mestizas en La Paz, Bolivia, viii + 170 pp.; La Paz: Praia.

TASSI, N., MEDEIROS, C., RODRIGUEZ-CARMONA, A. \& FERRUFINO, G., 2013 Hacer plata sin plata. El desborde de los comerciantes populares en Bolivia, xviii + 286 pp.; La Paz: PIEB. 